\title{
AVALIAÇÃO DAS FUNÇÕES COGNITIVAS, QUALIDADE DE SONO, TEMPO DE REAÇÃO E RISCO DE QUEDAS EM IDOSOS INSTITUCIONALIZADOS
}

Daianne Gonçalves ${ }^{1}$ Caroline Altermann²

Aline Vieira ${ }^{3}$

Ana Paula Machado ${ }^{4}$

Rachel Fernandes ${ }^{5}$

Andressa Oliveira ${ }^{6}$

Pámela Billig Mello-Carpes ${ }^{7}$ 
resumo

O processo de envelhecimento é acompanhado por uma série de alterações psicossociais e biológicas, dentre as quais está incluído o declínio cognitivo. Dependendo das condições genéticas, estilo de vida, atividades e interações sociais do idoso, o declínio pode ser acelerado ou retardado. O objetivo deste trabalho foi avaliar o perfil cognitivo, o tempo de reação, o risco de quedas e a qualidade do sono de idosos institucionalizados da cidade de Uruguaiana-RS. Foram avaliados 10 idosos de ambos os sexos utilizando como instrumentos o Mini-Exame do Estado Mental (MEEM), a Escala de Depressão Geriátrica (EDG), o Inventário de Ansiedade Traço-Estado, o Teste de Tempo de Reação (TTR), a Escala de Eficácia de Quedas (EEQ) e o Índice de Qualidade de Sono de Pittsburgh (IQSP). Os resultados apontaram a presença de declínio cognitivo em 40\% dos idosos, indícios de depressão em 60\% deles e tendência ao desenvolvimento de ansiedade. 76\% dos idosos classificaram a qualidade de seu sono como boa, mencionando alguns fatores que interferem na mesma, e 64\% revelaram haver preocupação ou medo de cair. No TTR os idosos apresentaram escore médio de 2,175 \pm 0,32 s. Estes resultados podem estar relacionados ao estilo de vida adotado pela maioria dos idosos institucionalizados, fora do seu convívio familiar, favorecendo seu isolamento e sua inatividade física e mental, gerando declínio da capacidade cognitiva. Nossos resultados demonstram um percentual significativo de risco de demência, depressão e/ou ansiedade nos idosos institucionalizados avaliados.

Palavras-chave

Envelhecimento. Institucionalização. Quedas. Cognição. Sono.

O crescimento da população mundial idosa é um fenômeno que vem ocorrendo nas últimas décadas com mais intensidade nos países em desenvolvimento, devido às melhorias nas condições de vida e saúde, como os avanços no tratamento e prevenção de doenças. Atualmente, já existem mais de 20 milhões de idosos no país, correspondendo a $10,8 \%$ da população total 
e estima-se que no ano de 2050, o número de idosos no Brasil ultrapasse os 64 milhões, representando mais de $22 \%$ da população (OMS, 2005; IBGE, 2010; IBGE, 2011).

No entanto, nem sempre o processo de envelhecimento é positivo, uma vez que pode trazer limitações funcionais e cognitivas, além de outras condições crônicas como a depressão, fazendo com que muitos idosos deixem de participar do ambiente social em que vivem, entregando-se à aposentadoria de maneira passiva, inativa e menos reflexiva, o que representa prejuízo a sua saúde mental e física, além de constituir fator de risco para o declínio cognitivo e para a demência (SOUZA; CHAVES, 2005; VALCARENGHI et al., 2011).

De acordo com Teixeira e Neri (2008), um envelhecimento bem-sucedido considera a existência de déficits cognitivos e fisiológicos associados à idade que são geneticamente determinados; assim, dentre as várias alterações fisiológicas decorrentes do processo de envelhecimento, as funções do Sistema Nervoso Central (SNC) - principalmente as de origem neuropsicológica envolvidas no processo cognitivo, tais como o aprendizado e a memória constituem um dos principais alvos de pesquisas realizadas sobre a senescência, já que estas alterações podem comprometer o bem-estar biopsicossocial do idoso, impedindo a continuidade da sua vida social e a interação com os familiares e a sociedade (SOUZA; CHAVES, 2005).

Embora boa parte da população idosa seja independente e resida na comunidade, alguns idosos precisam contar com o apoio de instituições residenciais de longa permanência. Essa parcela, na maioria das vezes, é constituída de idosos muito desvalidos, frequentemente abandonados pela família, acumulando problemas sociais e médicos.

As instituições, muitas vezes, não têm infra-estrutura adequada e profissionais qualificados para atender aos idosos, existindo também instituições clandestinas que constituem verdadeiros depósitos de velhos (MENDONÇA, 2006). Colaborando para este dado, pouquíssimos são os cursos oferecidos para formação de profissionais aptos a trabalhar com idosos. Considerando estas condições, é normal que a perda da função cognitiva dos idosos institucionalizados tenda a acelerar seu ritmo.

O envelhecimento traz consigo uma série de alterações que aumentam os fatores de risco e a ocorrência de doenças crônico-degenerativas (TORRES et al., 2009). O conjunto destas alterações pode gerar diminuição na capacidade funcional, aumento do risco de quedas e alterações no equilíbrio, no tempo de reação e no sono, podendo contribuir para o aumento da morbidade e mortalidade em idosos. 
Segundo Oliveira et al. (2010), os problemas com o sono ocorrem em mais da metade dos idosos com 65 anos de idade ou mais. A má qualidade do sono em idosos causa uma diminuição da capacidade física, aumentando a possibilidade de quedas frequentes; além de acarretar declínio cognitivo, esses fatores resultam em uma baixa qualidade de vida em indivíduos idosos (QUINHONES; GOMES, 2011).

Com o aumento da idade, as queixas relacionadas ao sono parecem acentuar-se (QUINHONES; GOMES, 2011). Alguns fatores contribuem para os problemas de sono e podem ser classificados em categorias: dor ou desconforto físico, fatores ambientais, desconfortos emocionais e alterações no padrão do sono. Essas alterações incluem aumento do período de tempo para o início do sono, dificuldade para reiniciar o sono e menor duração deste, além do aumento da fragmentação do mesmo e dos problemas respiratórios durante a noite. São também prevalentes a sonolência e a fadiga diurna, com aumento de cochilos, e o comprometimento cognitivo e do desempenho diurno (QUINHONES; GOMES, 2011; COSTA; CEOLIM; NERI, 2011; ZILLI; FICCA; SALZARULO, 2009).

O tempo de reação do idoso amplia-se, particularmente pelo fato de avaliar a capacidade de um indivíduo de responder com rapidez aos eventos ambientais, envolvendo fatores como a percepção e interpretação do estímulo e a programação e execução de uma resposta motora. A velocidade comportamental diminui com o envelhecimento e a lentidão torna-se significativa, pois afeta a maneira pela qual executam-se tarefas funcionais diárias. A velocidade comportamental reduzida em muitos cidadãos idosos pode estar relacionada com o aumento dos índices de acidentes e das taxas dos seguros de saúde, além da discriminação pela idade (SPIRDUSO, 2005; BINOTTO; FARIAS, 2009; ROSSATO; CONTREIRA; CORAZZA, 2011).

Pessoas de todas as faixas etárias apresentam risco de sofrer quedas, porém, para os idosos, elas possuem um significado muito relevante, pois podem levá-los à incapacidade, injúria e morte. Seu custo social é imenso e torna-se maior quando o idoso tem diminuição da autonomia e da independência ou passa a necessitar de institucionalização. Muitos idosos têm desafios no seu próprio ambiente domiciliar, e, geralmente, não caem por realizar atividades perigosas, e sim atividades rotineiras (VALCARENGHI et al., 2011).

Considerando estas questões, o objetivo deste trabalho foi avaliar as funções cognitivas, a qualidade de sono, o tempo de reação e o risco de quedas em idosos institucionalizados. 
A amostra utilizada foi composta por 10 idosos institucionalizados (7 mulheres e 3 homens), residentes em uma instituição particular voltada à classe de baixa renda do município de Uruguaiana-RS.

Foram critérios de inclusão: ter mais de 60 anos, residir na instituição asilar por um tempo mínimo de 3 meses e assinar um termo de consentimento livre e esclarecido, concordando em participar da pesquisa. Adotou-se como critério de exclusão a não realização completa das avaliações.

Este trabalho foi aprovado pelo Comitê de Ética em Pesquisa da UNIPAMPA (carta de aprovação 024/2010) em 10 de novembro de 2010.

\subsection{Procedimentos}

Após contato inicial para expor os objetivos do trabalho e conhecer as características da amostra, os idosos responderam a um formulário com o objetivo de traçar o seu perfil sociodemográfico e depois passaram pelas avaliações: cognitiva, de sono, de risco de quedas e de tempo de reação. Os instrumentos de pesquisa utilizados estão listados a seguir.

Mini-exame do estado Mental (MEEM): dividido em 5 sessões, avalia orientação, memória imediata, atenção e cálculo, evocação e linguagem (FOLSTEIN; FOLSTEIN; MCHUGH,, 1975; BRUCKI et al., 2003), permitindo uma avaliação das funções cognitivas;

Escala de Depressão Geriátrica (GDS): é utilizada para detectar a percepção de depressão em idosos, oferecendo mensurações válidas e confiáveis para avaliar transtornos depressivos. É composta por 30 perguntas com 2 possibilidades de resposta, sim e não (YESAVAGE, 1983). Adotou-se ponto de corte com escore de 0 a 10 para ausência de depressão, de 11 a 20 para depressão moderada e de 21 a 30 para depressão grave;

Inventário de Ansiedade Traço-Estado (IDATE): trata-se de um questionário de autoavaliação dividido em duas partes. A primeira avalia a ansiedade-traço e a segunda avalia a ansiedade-estado, sendo que cada parte contém 20 afirmações. O questionário possui uma escala de 4 itens que variam de 1 a 4, sendo que ESTADO significa como o sujeito se sente no "momento" e o TRAÇO como ele "geralmente se sente". O escore de cada parte varia de 20 a 80 pontos, sendo que, quanto mais baixo o escore, menor é o grau de ansiedade (SPIELBERGER; GORSUCH; LUSHENE, 1970; ANDREATINI; SEABRA, 1993). 
Teste de Tempo de Reação (TTR): determina o intervalo de tempo que decorre entre a apresentação de um estímulo até o início da resposta da pessoa. Para esta avaliação o idoso foi colocado em frente a um computador portátil e orientado a pressionar a tecla de espaço utilizando a sua mão preferida cada vez que um estímulo visual (círculo que aparecia na tela do computador) mudasse de cor. O procedimento foi repetido três vezes e utilizou-se a média das três tentativas (CORAZZA; PEREIRA; DIAS, 2007; SCHMIDT; WRISBERG, 2001; ROSSATO; CONTREIRA; CORAZZA, 2011).

Índice de Qualidade do Sono de Pittsburgh (IQSP): avalia a qualidade do sono durante os últimos 30 dias (BUYSSE et al., 1989; CORRÊA; CEOLIM, 2008).

Escala de Eficácia de Quedas (EEQ): utilizada para mensurar o medo de cair ao realizar 16 atividades, incluindo as atividades de vida diárias (atender ao telefone, limpar a casa, tomar banho), atividades externas (ir às compras, caminhar pela vizinhança) e de participação social (visitar amigos ou parentes, ir a uma atividade social). As alternativas de resposta variam de 1 a 4 pontos e o escore de 16 a 64 pontos, sendo que 16 pontos corresponde à ausência de preocupação e 64 à preocupação extrema em relação a quedas (HAUER et al., 2010; LOPES et al., 2009).

Os dados foram analisados estatisticamente através do programa Microsoft Excel for Windows e estão expressos na forma de média \pm desvio padrão e/ou percentuais.

\section{Resultados}

O grupo de idosos participantes da pesquisa foi constituído por 7 mulheres e 3 homens. A idade média da amostra foi $79,5( \pm 10,7)$ anos, sendo que o mais novo tinha 60 anos e o mais velho 94 anos. O tempo de institucionalização variou de 3 a $57(23,1 \pm 20,7)$ meses. Dos 10 idosos, 2 não eram alfabetizados e os demais possuíam maior nível de escolaridade, ou seja, sabiam ler, o que foi levado em consideração na análise final dos resultados (visto que o MEEM leva em consideração esta informação para definir a presença de declínio cognitivo enquanto os demais testes não requerem capacidade de leitura). 
De acordo com o resultado final do MEEM, $40 \%$ dos idosos avaliados foram classificados com possível demência. A média final da amostra no exame foi $17,7( \pm 6,2)$. Na tabela 1 são apresentados os resultados obtidos nas diferentes domínios do MEEM.

Tabela 1 - Resultados obtidos nos diferentes instrumentos de avaliação.

\begin{tabular}{l|c}
\hline \multicolumn{1}{c|}{ Instrumento } & Escore obtido (média \pm DP) \\
\hline MEEM - Orientação (0-10 pontos) & $6,1 \pm 3,4$ \\
\hline MEEM - Registro (0-3 pontos) & $1,9 \pm 1,4$ \\
\hline MEEM - Atenção (0-5 pontos) & $1,1 \pm 1,4$ \\
\hline MEEM - Evocação (0-3 pontos) & $0,8 \pm 1,1$ \\
\hline MEEM - Linguagem (0-9 pontos) & $7,8 \pm 1,0$ \\
\hline MEEM - Escore final (0-30 pontos) & $17,7 \pm 6,2$ \\
\hline Escala de depressão geriátrica (0-30 pontos) & $12,9 \pm 7,0$ \\
\hline IDATE traço (20-80 pontos) & $52,6 \pm 8,6$ \\
\hline IDATE estado (20-80 pontos) & $44 \pm 4,8$ \\
\hline
\end{tabular}

Na avaliação da depressão através da GDS, o escore médio foi 12,9 ( \pm $7,0)$, média que indica a presença de quadro depressivo leve. Na classificação individual, 6 dos 10 idosos (60\%) se enquadraram em possível quadro depressivo segundo este instrumento.

A média da amostra no IDATE Traço foi de $52,6( \pm 8,6)$ e no IDATE Estado $44( \pm 4,8)$. Esses valores intermediários revelam tendência à ansiedade nos idosos participantes.

Na IQSP, verificamos que os idosos dormem por volta das 19,9h $( \pm 0,8)$, levando aproximadamente $1,4 \mathrm{~h}( \pm 1,0)$ para dormir, acordam em média às $7,3 \mathrm{~h}( \pm 0,8)$ da manhã e dormem $10,2 \mathrm{~h}( \pm 2,0)$ de sono por noite. A percepção da qualidade de sono foi classificada pelo próprio idoso: $76 \%$ a classificaram como boa, somente $12 \%$ a classificaram como muito boa e o mesmo percentual como ruim. Entretanto, identificamos muitos distúrbios de sono neste grupo de idosos (tabela 2). 
Tabela 2 - Principais causas de distúrbio de sono nos idosos avaliados segundo o IQSP.

\begin{tabular}{l|c}
\hline \multicolumn{1}{c|}{ Componentes do IQSP } & Porcentagem de idosos \\
\hline Demora mais de 30 min. para dormir & $70,83 \%$ \\
\hline Acorda no meio da noite & $66,60 \%$ \\
\hline Levanta para ir ao banheiro & $58,33 \%$ \\
\hline Dificuldade de respirar & $12,50 \%$ \\
\hline Tosse ou ronca & $58,33 \%$ \\
\hline Sente frio & $75,00 \%$ \\
\hline Sente calor & $20,83 \%$ \\
\hline Pesadelos & $16,66 \%$ \\
\hline Dores & $54,16 \%$ \\
\hline Outra razão & $12,5 \%$ \\
\hline Toma medicamento para dormir & $33,33 \%$ \\
\hline Problema em ficar acordado durante o dia & $16,66 \%$ \\
\hline
\end{tabular}

No TTR, que mediu o tempo de reação motora em resposta a um estímulo visual, o escore final médio foi de $2,175 \pm 0,32 \mathrm{~s}$. A fim de relacionar os resultados obtidos no TTR com a função cognitiva, utilizou-se estatística descritiva (Teste de Correlação de Pearson). A correlação encontrada foi forte ( $R=-0,72)$, o que parece estar diretamente relacionado com o baixo nível de atividades cognitivas realizadas pelos idosos avaliados, evidenciado nos baixos escores do MEEM já que o TTR envolve a função cognitiva para a programação da resposta motora, por mais simples que esta seja.

A preocupação com a possibilidade de quedas realizando atividades cotidianas em geral, avaliada através da EEQ, apresentou média de 31,75 pontos, o que revela medo de cair. Acredita-se que possa haver relação entre a preocupação com a possibilidade de sofrer quedas e o declínio das atividades de interação social, de prática de atividades físicas e de vida diária.

\section{Discussão}

Verificamos que $40 \%$ dos idosos institucionalizados avaliados apresentaram risco de demência de acordo com o MEEM e 60\%, indícios de depressão (EGD). À medida que a idade avança, existe uma progressiva 
perda de recursos físicos, mentais e sociais, a qual tende a despertar sentimentos de desamparo (DAVIM et al., 2004). A velhice parece deixar o indivíduo impotente, indefeso e fragilizado para tomar suas próprias decisões, para enfrentar seus problemas e o cotidiano, não só diante dos familiares, mas também da sociedade como um todo. Sendo assim, o idoso tem sido visto como uma pessoa improdutiva, ultrapassada, e pouco se tem feito para recuperar sua identidade e elevar sua autoestima (BEUTER et al., 2012; FARBER, 2012), o que pode contribuir para o surgimento de depressão e do próprio declínio cognitivo.

As mudanças experimentadas pelo idoso, como a perda do companheiro (a), as doenças, a dependência física, a institucionalização e o distanciamento de seu espaço familiar, podem ser o ponto de partida para uma desestruturação psíquica. É nesse contexto que a depressão surge como um dos mais importantes danos à saúde da terceira idade.

Corrêa e Ceolim (2008) afirmam, em relação à qualidade de sono, que há um aumento no número de indivíduos afetados diretamente proporcional ao aumento da idade. Alonso e Estebaranz (2006) acrescentam que pessoas idosas manifestam dificuldades em obter um grau satisfatório de sono, destacando a importância da avaliação do mesmo ao avaliar a saúde do idoso. Embora a literatura aponte para uma redução da qualidade e quantidade do sono com o envelhecimento normal (ARAÚJO; CEOLIM, 2010; QUINHONES; GOMES, 2011; COSTA; CEOLIM; NERI, 2011), há idosos satisfeitos com seu padrão de sono, como constatado em nosso estudo e no de Oliveira et al. (2010). Os dados encontrados na realização desse estudo revelaram que apenas $12 \%$ dos indivíduos avaliados classificaram sua qualidade de sono como ruim, a maior parte (76\%) a classificou como boa e os outros $12 \%$ como muito boa, porém, simultaneamente, fora identificada uma série de aspectos que prejudicam ou causam distúrbios do sono.

Para pessoas acima de 60 anos, de acordo com Rossato, Contreira e Corazza (2011) e Spirduso (2005), o resultado no teste de tempo de reação simples apresenta valores satisfatórios entre $340 \mathrm{~ms}$ e $380 \mathrm{~ms}(0,34$ e 0,38 segundos), sendo que os autores observaram que homens após 60 anos reagem a estímulos simples mais rapidamente que mulheres. $\mathrm{O}$ resultado por nós encontrado nesse estudo $(2,175 \pm 0,32 \mathrm{~s})$ apresenta-se distante do valor satisfatório, fato que pode estar relacionado com o processo de institucionalização, a diminuição das funções cognitivas (conforme verificado no teste de correlação com o MEEM), a não realização de atividades físicas por estes idosos ou, simplesmente, com a dificuldade dos idosos em realizar esta 
tarefa, uma vez que a maioria nunca tinha tido acesso a um computador, e isto também deve ser considerado.

O envelhecimento causa alterações substanciais na capacidade de processamento de informações sensoriais em idosos e diminuição significativa das respostas reflexas e reações rápidas. Desta forma, a detecção de um estímulo fica prejudicada, tornando as respostas motoras mais lentas (CÔRTES; GAMA; DIAS, 2007). Cabe ressaltar que o tempo de reação é especialmente importante no sujeito idoso pois, à medida que o tempo entre perceber uma situação e responder a ela se torna menor, o idoso tende a ter maior capacidade de responder diante de uma situação da vida diária, por exemplo, um desequilíbrio, reduzindo, assim, o risco de sofrer uma queda.

A possibilidade de quedas entre os idosos é reconhecida como um importante problema de saúde pública, em decorrência da frequência, da morbidade e do elevado custo social e econômico decorrente das lesões provocadas, como afirmam Binotto e Farias (2009), além de afetarem as relações de convívio e interação dos mesmos. Os idosos avaliados aqui revelaram medo de cair realizando desde tarefas que envolvem ambientes externos e maior atenção, como subir escadas, até atividades antes consideradas simples, como tomar banho, mas que com a senilidade começam a apresentar grau de dificuldade.

Em estudos realizados por Cress et al. (2004) e Christofoletti et al. (2006), o declínio cognitivo é apontado como uma variável que influencia diretamente o risco de quedas em idosos. Além do declínio cognitivo, a diminuição das capacidades visual e espacial, da audição e do equilíbrio, somados a fatores externos, como má conservação de calçadas e vias públicas, falta de adaptação e adequação do ambiente de moradia (como a utilização de barras de apoio e piso antiderrapante) também são fatores que aumentam substancialmente a susceptibilidade de quedas nesta faixa etária.

Dessa forma, levando-se em consideração a elevação da longevidade da população geriátrica do Brasil (e do mundo), é necessário um olhar atencioso ao idoso, uma vez que a Política Nacional de Saúde do Idoso (PNSI), sancionada em 1999, assegura aos idosos: a promoção do envelhecimento saudável; a manutenção da capacidade funcional; a assistência às necessidades de saúde do idoso; a reabilitação da capacidade funcional comprometida; a capacitação de recursos humanos especializados; o apoio ao desenvolvimento de cuidados informais e o apoio a estudos e pesquisas (BRASIL, 1999).

A estimulação da cognição, através de atividades que exercitam o raciocínio e a memória, bem como boas práticas de saúde - como alimentação saudável e exercícios -, apesar de parecerem simples, somam ganhos 
significativos para os idosos, uma vez que estes devem permanecer mental e fisicamente ativos para um processo de envelhecimento sadio e com preservação da qualidade de vida.

\section{Considerações finais}

Os dados apresentados nesse estudo demonstram, de forma geral, a situação cognitiva de um grupo de idosos institucionalizados. Um percentual significativo dos idosos avaliados apresentou risco de demência, depressão e/ ou ansiedade. Além disso, os idosos apresentaram, apesar de uma percepção positiva de sua qualidade de sono, distúrbios associados ao sono. O risco de quedas e o tempo de reação foram altos, podendo estar ambos relacionados aos resultados obtidos na avaliação cognitiva.

Estes resultados apontam para a necessidade de um olhar mais cuidadoso das famílias, da sociedade e das políticas públicas para estas instituições, no sentido de que sejam garantidas ações que permitam a manutenção de uma boa qualidade de vida dos idosos institucionalizados. A promoção de atividades físicas e cognitivas parece ser uma alternativa não muito onerosa e bem documentada que pode proporcionar estes benefícios.

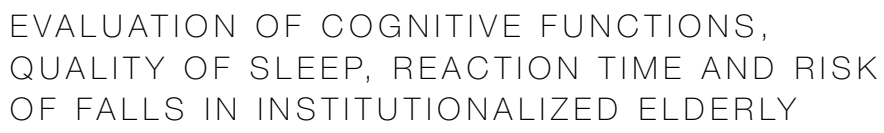

\section{abstract}

The aging process is accompanied by a series of biological and psychosocial changes, among which is included cognitive decline. Depending on genetics, conditions of life, activities and social interactions of the elderly, the decline can be accelerated or decelerated. The objective of this work was evaluate the cognitive profile, the reaction time, the risk of falls and the quality of sleep of institutionalized elderly of Uruguaiana-RS. We evaluated 10 elderly of both sexes using as instruments the Mini-Mental State Examination, the Geriatric Depression Scale, the State-Trait Anxiety Inventory, the Reaction Time Test, the Falls Efficacy Scale and the Pittsburgh Sleep Quality Index. The results indicated presence of cognitive decline in $40 \%$ of elderly, signs of depression in $60 \%$ and tendency to the development of anxiety. $76 \%$ of elderly rated their quality of sleep as 

time score the elderly showed a mean of $2.175 \pm 0.32 \mathrm{~s}$. These results might be related to the lifestyle adopted by most of the institutionalized elderly, out of their family context, often contributing to their isolation and their physical/mental inactivity, generating cognitive decline. Our results demonstrate a significant percentage of risk of dementia, depression and/or anxiety in evaluated institutionalizated elderly.

keywords

Aging. Institutionalization. Falls. Cognition. Sleep.

\author{
referências
}

ALONSO, Concepcion Aparicio; ESTEBARANZ, Ana Isabel Mejía. Sleeping difficulties among the elderly. Revista de Enfermeria, Barcelona, v. 29, n. 3, p. 48-52, marzo. 2006.

ANDREATINI, Roberto; SEABRA, Maria de Lourdes. A estabilidade do IDATE-traço: avaliação após cinco anos. Revista da Associação Brasileira de Psiquiatria - Asociación Psiquiatrica de la America Latina, São Paulo, v. 15, n. 1, p. 21-25, jan./mar. 1993.

ARAÚJO, Claudia Lysia de Oliveira; CEOLIM, Maria Filomena. Qualidade do sono de idosos residentes em instituição de longa permanência. Revista da Escola de Enfermagem da USP, São Paulo, v. 44, n. 3, p. 619-626, set. 2010.

BEUTER, Margrid et al. Sentimentos de familiares acompanhantes de adultos face ao processo de hospitalização. Escola Anna Nery, Rio de Janeiro, v. 16, n. 1, p. 134-140, jan./mar. 2012

BINOTTO, Maria Angélica; FARIAS, Sidney Ferreira. A relação entre tempo de reação e o acometimento por quedas em idosas. In: CONGRESSO BRASILEIRO DE CIÊNCIAS DO ESPORTE E CONGRESSO INTERNACIONAL DE CIENCIAS DO ESPORTE, 16. 2009, Salvador. Anais... Salvador: SOAC, 2009. p. 1-6.

BRASIL. Política Nacional do Idoso, Portaria no 1.395, de 9 de dezembro de 1999. Brasília: Diário Oficial [da] República Federativa do Brasil, Poder Executivo, Brasília, DF, 13 dez. 1999. Seção 1, p. 20-24.

BRUCKI, Sonia et al. Sugestões para o uso do mini-exame do estado mental no Brasil. Arquivo de Neuropsiquiatria, São Paulo, v. 61, n. 3B, p. 777-781, set. 2003

BUYSSE, Daniel et al. The Pittsburgh sleep quality index: a new instrument for psychiatric practice and research. Psychiatry Research, Amsterdam, v. 28, n. 2, p. 193-213, May 1989

CHRISTOFOLETTI, Gustavo et al. Risco de quedas em idosos com doença de Parkinson e demência de Alzheimer: um estudo transversal. Revista Brasileira de Fisioterapia, São Carlos, v. 10, n. 4, p. 429-433, out./dez. 2006. 
CORAZZA, Sara Terezinha; PEREIRA, Érico Felden; DIAS, Jonathan Ache. Creation, development and analysis of reproductiveness of test to evaluate simple and choice reaction times. The FIEP Bulletin, v. 77, n. 1, p. 613-615, 2007.

CORREAA, Karina; CEOLIM, Maria Filomena. Qualidade do sono em pacientes idosos com patologias vasculares periféricas. Revista da Escola de Enfermagem da USP, São Paulo, v. 42, n. 1, p. 12-18, 2008.

CÔRTES, Guilherme Gomes; GAMA, Júlia de Fátima Ribeiro; DIAS, André Gonçalves, Processamento mental e tempo de reação em mulheres idosas ativas e praticantes de treinamento contra-resistência em comparação com idosas sedentárias e mulheres jovens. Vértices, Rio de Janeiro, v. 9, n. 1, p. 51-60, jan./dez. 2007.

COSTA, Shintia Viana da; CEOLIM, Maria Filomena; NERI, Anita Liberalesso. Problemas de sono e suporte social: estudo multicêntrico Fragilidade em Idosos Brasileiros. Revista Latino-Americana de Enfermagem, São Paulo, v. 19, n. 4, p. 1-8, jul./ago. 2011.

CRESS, Elaine et al. Physical activity programs and behavior counseling in older adult population. Medicine and Science in Sports and Exercise, Hagerstown, v. 36, n. 11, p. 1997-2003, Oct. 2004.

DAVIM, Rejane Marie Barbosa et al. Estudo com idosos de instituições asilares no município de Natal/RN: características socioeconômicas e de saúde. Revista Latino-Americana Enfermagem, Ribeirão Preto, v. 12, n. 3, p. 518-524, maio/jun. 2004.

FARBER, Sonia. Envelhecimento do corpo: noções díspares nas mídias atuais. Revista Comunicação e Sociedade, Portugal, v. 21, p. 123-133, 2012.

FOLSTEIN, Marshal; FOLSTEIN, Susan; McHUGH, Paul. "Mini-Mental State": a practical method for grading the cognitive state of patients for the clinical. Journal of Psychiatry Research, Philadelphia, v. 12, n. 3, p. 189-198, Nov. 1975.

HAUER, Klaus et al. Validation of the Falls Efficacy Scale and Falls Efficacy Scale International in Geriatric Patients with and without Cognitive Impairment: Results of Self-Report and Interview-Based Questionnaires. Gerontology, Basel, v. 56, n. 2, p. 190-199, Sept. 2010.

IBGE - INSTITUTO BRASILEIRO DE GEOGRAFIA E ESTATÍSTICA. Censo demográfico 2010: características da população e dos domicílios - resultados do universo. Rio de Janeiro: IBGE, 2011.

. Sintese de indicadores sociais: uma análise das condições de vida da população brasileira 2010. Rio de Janeiro: IBGE, 2010.

LOPES, Kedma Teixeira et al. Prevalência do medo de cair em uma população de idosos da comunidade e sua correlação com mobilidade, equilíbrio dinâmico, risco e histórico de quedas. Revista Brasileira de Fisioterapia, São Carlos, v. 13, n. 3, p. 223-229, maio/jun. 2009.

MENDONÇA, Jurilza Maria Barros de. Instituição de longa permanência para idosos e políticas públicas. Revista Kairós, São Paulo, v. 9, n. 2, p. 169-190, dez. 2006.

OMS - Organização Mundial da Saúde. Envelhecimento Ativo: uma Política de Saúde. Brasilia: Organização Pan-Americana da Saúde, 2005.

OLIVEIRA, Beatriz Helena Domingos et al. Relações entre padrão do sono, saúde percebida e variáveis socioeconômicas em uma amostra de idosos residentes na comunidade: Estudo PENSA. Ciência \& Saúde Coletiva, Rio de Janeiro, v. 15, n. 3, p. 851-860, maio 2010. 
QUINHONES, Marcos Schmidt; GOMES, Marleide da Mota. Sono no envelhecimento normal e patológico: aspectos clínicos e fisiopatológicos. Revista Brasileira de Neurologia, Rio de Janeiro, v. 47, n. 1, p. 31-42, jan./mar. 2011.

ROSSATO, Luana Callegaro; CONTREIRA, Andressa Ribeiro; CORAZZA, Sara Teresinha. Análise do tempo de reação e do estado cognitivo em idosas praticantes de atividades físicas. Fisioterapia e Pesquisa, São Paulo, v. 18, n. 1, p. 54-59, jan./mar. 2011.

SCHMIDT, Richard; WRISBERG, Craig. Aprendizagem e performance motora: uma abordagem da aprendizagem baseada no problema. Porto Alegre: Artmed, 2001.

SOUZA, Juliana Nery de; CHAVES, Eliane Corrêa. $O$ efeito do exercício de estimulação da memória em idosos saudáveis. Revista da Escola de Enfermagem da USP, São Paulo, v. 39, n. 1, p. 13-19, mar. 2005.

SPIELBERGER, Charles; GORSUCH, Richard; LUSHENE, Robert. Manual for the State-Trait Anxiety Inventory. Palo Alto: Consulting Psychologists Press, 1970

SPIRDUSO, Waneen Wyrick. Dimensões físicas do envelhecimento. São Paulo: Manole, 2005.

STOPPE JÚNIOR, Alberto; JACOB FILHO, Wilson; LOUZA NETO, Mario. Avaliação de depressão em idosos através da Escala de Depressão em Geriátrica: resultados preliminares. Revista da Associação Brasileira de Psiquiatria - Asociación Psiquiatrica de la America Latina, São Paulo, v. 16, n. 4, p. 149-153, out./dez. 1994.

TEIXEIRA, IIka Nicéia D'Aquino Oliveira; NERI, Anita Liberalesso. Envelhecimento bem-sucedido: uma meta no curso da vida. Psicologia USP, São Paulo, v. 19, n. 1, p. 81-94, jan./mar. 2008.

TORRES, Gilson de Vasconcelos et al. Avaliação da capacidade de realização das atividades cotidianas em idosos residentes em domicílio. Revista Baiana, Salvador, v. 33, n. 3, p. 466-475, jul./set. 2009.

VALCARENGHI, Rafaela Vivian et al. Alterações na funcionalidade/cognição e depressão em idosos institucionalizados que sofreram quedas. Acta Paulista de Enfermagem, São Paulo, v. 24, n. 6, p. 828-833, 2011

YESAVAGE, Jerome A. et al. Development and validation of a geriatric depression screening scale: a preliminary report. Journal of Psychiatric Research, Great Britain, v. 17, n. 1, p. 37-49, 1983.

ZILLI, Iole; FICCA, Gianluca; SALZARULO, Piero. Factors involved in sleep satisfaction in the elderly. Sleep Medicine, v.10, n. 2, p. 233-239, Feb. 2009 